Serrano García de Dionisio, F.; Gómez-Carmona, C.D.; Bastida-Castillo, A.; Rojas-Valverde, D.; Pino-Ortega, J. (2020) Slope Influence on the Trail Runner Physical Load: A Case Study. Revista Internacional de Medicina y Ciencias de la Actividad Física y el Deporte vol. 20 (80) pp. 641-658 Http://cdeporte.rediris.es/revista/revista80/artinfluencia1191.htm

DOI: https://doi.org/10.15366/rimcafd2020.80.012

\title{
ORIGINAL
}

\section{INFLUENCIA DEL DESNIVEL EN LA CARGA FÍSICA DEL CORREDOR DE MONTAÑA: UN ESTUDIO DE CASO}

\section{SLOPE INFLUENCE ON THE TRAIL RUNNER PHYSICAL LOAD: A CASE STUDY}

Serrano García de Dionisio, F." ; Gómez-Carmona, C.D. ${ }^{2}$; Bastida-Castillo, A. ${ }^{3}$; Rojas-Valverde, D. ${ }^{4,5}$ y Pino-Ortega, J. ${ }^{6}$

${ }^{1}$ Graduado en Ciencias de la Actividad Física y el Deporte. Facultad de Ciencias del Deporte, Universidad de Murcia (España) fsgd92@gmail.com

2 Personal investigador con contrato predoctoral en Grupo de Optimización del Entrenamiento y el Rendimiento Deportivo (GOERD). Departamento de Didáctica de la Expresión Musical, Plástica y Corporal. Facultad de Ciencias del Deporte, Universidad de Extremadura (España) cdgomezcarmona@unex.es

${ }^{3}$ Doctor en Ciencias del Deporte. Departamento de Actividad Física y Deporte. Facultad de Ciencias del Deporte, Universidad de Murcia, España. Profesor Asociado Universidad Isabel I, Burgos (España) alejandro.bastida@um.es

${ }^{4}$ Estudiante de Doctorado, Grupo de Avances en Entrenamiento Deportivo y Acondicionamiento Físico (GAEDAF), Facultad de Ciencias del Deporte, Universidad de Extremadura, Cáceres (España) 5 Investigador Asociado, Centro de Investigación y Diagnóstico en Salud y Deporte (CIDISAD). Profesor en Escuela de Ciencias del Movimiento Humano y Calidad de Vida. Universidad Nacional de Costa Rica (Costa Rica) drojasv@una.cr

${ }^{6}$ Doctor en Ciencias de la Actividad Física y el Deporte. Profesor en Facultad de Ciencias de la Actividad Física y del Deporte. Departamento de Actividad Física y Deporte. Universidad de Murcia (España) josepinoortega@um.es

\section{FINANCIACIÓN}

Para la realización de este estudio no se ha recibido ninguna ayuda por parte de la empresa encargada del desarrollo y comercialización de la tecnología utilizada en esta investigación. El autor Carlos D. Gómez Carmona es beneficiario de una beca del Ministerio de Ciencia, Innovación y Universidades (FPU17/00407).

Código UNESCO / UNESCO code: 5899 Otras especialidades pedagógicas (Educación Física y Deporte).

Clasificación Consejo de Europa / Council of Europe Classification: 17. OTRAS (Análisis de las exigencias deportivas) / OTHER (Sport demands analysis) 
Recibido 17 de noviembre de 2018 Received November 17, 2018 Aceptado 4 de agosto de 2019 Accepted August 4, 2019

\section{RESUMEN}

Este estudio analizó las exigencias de carga interna (Frecuencia cardíaca, FC) y externa (Velocidad; Player Load, PL; Power Metabolic, PM; Stiffness Vertical, KVERT; entropía aproximada, ApEn) producidas en función del desnivel del terreno en una carrera de montaña. Un atleta de nivel nacional (edad: 25.3 años; altura: $172 \mathrm{~cm}$; peso: $67 \mathrm{~kg}$; $\mathrm{VO}_{2 \text { MAx: }} 70.2 \mathrm{ml} / \mathrm{kg} / \mathrm{min}$ ) participó en una prueba oficial (Distancia: 27.6 $\mathrm{km}$; Desnivel acumulado: $973 \mathrm{~m}$ ), siendo analizado en 6 segmentos respecto al desnivel (sin desnivel, positivo y negativo). El registro de datos se realizó mediante un dispositivo inercial WIMU PRO ${ }^{\text {TM }}$ (RealTrack Systems, Almería, España). Los resultados muestran un aumento de $\mathrm{PL} / \mathrm{min}$ y $\mathrm{PM} / \mathrm{min}$ en desnivel negativo y de $\mathrm{FC}_{\text {AVG }}$ en desnivel positivo. KVERT y ApEn fueron más bajos en desnivel positivo, mientras que la velocidad fue mayor sin desnivel. Se encuentra gran variabilidad en las exigencias en función de la orografía del terreno, siendo importante su análisis para el diseño específico del entrenamiento y la planificación del evento.

PALABRAS CLAVE: Carreras de montaña, dispositivos inerciales, frecuencia cardiaca, stiffness vertical, carga neuromuscular, fatiga.

\section{ABSTRACT}

This study analysed the internal (heart rate, HR) and external load demands (Speed; Player Load, PL; Power Metabolic, PM; Vertical stiffness, KVERT; approximated entropy, $\mathrm{ApEn}$ ) during a trail running race in relation to the slope. A national-level athlete (age: 25.3 years; height: $172 \mathrm{~cm}$; weight: $67 \mathrm{~kg}$; $\mathrm{VO}_{2 \mathrm{MAx}}: 70.2$ $\mathrm{ml} / \mathrm{kg} / \mathrm{min}$ ) participated in an official race (Distance: $27.6 \mathrm{~km}$; Accumulated slope: $973 \mathrm{~m}$ ), analysed in 6 segments related to the slope (without, positive and negative slope). Data was registered through an inertial device WIMU PRO ${ }^{\text {TM }}$ (RealTrack Systems, Almería, Spain). The results show an increase of $\mathrm{PL} / \mathrm{min}$ and $\mathrm{PM} / \mathrm{min}$ in negative slope and an increase of $\mathrm{HR}_{\mathrm{AVG}}$ in positive slope. $\mathrm{K}_{\mathrm{VERT}}$ and ApEn were lower in positive slope, while velocity was faster without slope. It is found a great variability in the trail runner demands related to terrain orography, being important its analysis to design specific training sessions and race planning.

KEYWORDS: Mountain running, inertial devices, heart rate, vertical stiffness, neuromuscular load, fatigue.

\section{INTRODUCCIÓN}

Dentro del fenómeno social running se encuentran una diversidad de pruebas atléticas de resistencia entre las que las disciplinas más practicadas son las carreras 
en ruta (medio maratón y maratón), las carreras de montaña (gran diversidad de terreno y distancias que tienen en común desniveles del terreno) y las carreras de ultra resistencia (por tiempo o por distancia siendo de distintas tipologías: a) senderos, b) dentro de un circuito visible y c) carreras por ciudad) (Consejo Superior de Deportes, 2016). Este tipo de competiciones cuenta con una historia muy reciente comparado a otras disciplinas deportivas y ha evolucionado con el tiempo de manera acelerada (Rojas-Valverde, 2019) y que tiene cada vez un mayor número de adeptos ya que, como reconocen los propios corredores, suponen un reto personal y les proporcionan un aumento del reconocimiento social de su grupo de iguales (Ruiz-Juan y Zarauz, 2014).

La especialidad de ultra fondo es una de las actividades más estresantes en las que un ser humano puede participar de forma voluntaria (Eich y Metcalfe, 2009), debido a la intensidad, la duración y condiciones ambientales que pueden ser adversas y cambiantes, que requieren del deportista un alto grado de preparación física específica y un enorme esfuerzo tanto físico (Gutiérrez-Vargas et al., 2020; Millet, 2011) como psicológico (Buceta, de la Llave, Pérez Llantada, Vallejo y del Pino, 2002).

La programación y monitorización del entrenamiento es clave para la obtención de rendimiento, así como para evitar la aparición de diferentes lesiones (Halson, 2014). La dificultad de la modalidad de las carreras de montaña pasa, precisamente, por la cuantificación para planificar de forma correcta el entrenamiento y la competición (Kerhervé, Millet, y Solomon, 2015). Esto se debe, fundamentalmente, a la dificultad que implica la orografía en la que se practica, ya que en un terreno irregular un mismo valor puede ser el resultado de diferentes factores.

La cuantificación de la carga está dividida en dos grandes grupos, carga externa y carga interna (Halson, 2014). Entre los que miden la carga externa encontramos los que cuantifican la potencia de salida (Jobson, Passfield, Atkinson, Barton, y Scarf, 2009), sistemas de análisis del movimiento donde se determinan valores mediante un sistema de medición inercial con sensores de posicionamiento satelital (Taylor, Chapman, Cronin, Newton, y Gill, 2012) y medidas derivadas del análisis de la fatiga neuromuscular entre los que destacan el stiffness o fuerzas de reacción durante la carrera (Kvert) y la carga neuromuscular (Cormack, Mooney, Morgan, y McGuigan, 2013; Twist y Highton, 2013).

Recientes investigaciones han determinado la fatiga neuromuscular como la pérdida de fuerza después de un ejercicio prolongado, provocando alteraciones en el patrón de carrera (Millet, 2011; Millet, Martin, Lattier, y Ballay, 2003; Millet et al., 2011). Diferentes trabajos han analizado el patrón de la carrera durante maratones (Kyröläinen et al., 2000; Martin et al., 2010; Nicol, Komi, y Marconnet, 2007) o ultramaratones (Millet, 2011; Millet et al., 2011; Morin, Tomazin, Edouard, y Millet, 2011). Nicol, Komi, y Marconnet (2007) no encontraron cambios en los parámetros cinemáticos del movimiento en maratón mientras que Kyröläinen et al (2000) reportó de forma significativa un incremento en la frecuencia de zancada. En cambio en 
ultramaratón, diferentes investigadores han evidenciado cambios en la mecánica de carrera teniendo una mayor frecuencia de zancada y reduciendo la fuerza de reacción vertical en la pisada y la amplitud de la oscilación del centro de masas, con el objetivo de ser más eficientes (Millet, 2011; Millet et al., 2009; Morin, Samozino, y Millet, 2011).

Por otro lado, entre aquellos que miden la carga interna encontramos percepción subjetiva del esfuerzo (RPE) (Borresen y Lambert, 2009); la frecuencia cardiaca $(\mathrm{HR})$ y la relación lineal de esta con el consumo máximo de oxígeno $\left(\mathrm{VO}_{2 \mathrm{MAX}}\right)$ (Uth, Sorensen, Overgaard, y Pedersen, 2004); índice de calidad del entrenamiento (TRIMP) (Banister, 1991); el nivel de lactato la cual es sensible a la intensidad y duración del ejercicio (Hughson, Weisiger, y Swanson, 1987); variabilidad de la HR en reposo o post-ejercicio para determinar si el sujeto se adapta o no a los estímulos proporcionados (Pichot et al., 2000), alteraciones mecánicas del músculo (Gutiérrez-Vargas et al., 2020), cambios en la temperatura de la piel como reflejo de la actividad metabólica (Gutiérrez-Vargas et al., 2017) y análisis de parámetros bioquímicos (Gutiérrez-Vargas et al., 2020; Urdampilleta, López-Grueso, MartínezSanz y Mielgo-Ayuso, 2014).

Para el análisis de estas variables, dispositivos inerciales han sido desarrollados en los últimos años los cuáles sincronizan en el tiempo datos de carga interna y externa a partir de los diferentes sensores de los que disponen (acelerómetros, giróscopos, magnetómetros, GPS, receptores de dispositivos externos mediante tecnología bluetooth y Ant+, entre otros) para su análisis en tiempo real o a posteriori (Gabbett, 2013). Además, han sido ampliamente utilizados en la literatura para analizar tanto movimientos cíclicos (Nedergaard et al., 2017) como deportes colectivos (Boyd, Ball, y Aughey, 2013; Cormack et al., 2013) reportando una alta fiabilidad y validez (Barrett, Midgley, y Lovell, 2014).

Teniendo en cuenta las dificultades que genera la cuantificación de la carga de las carreras de montaña y la necesidad de una correcta planificación para conseguir el máximo rendimiento evitando el sobreentrenamiento, los objetivos de este estudio son: (a) conocer las exigencias de carga interna y externa que se producen durante una prueba de competición oficial de carrera de montaña y (b) determinar si la orografía es un factor que provoca alteraciones en los distintos parámetros analizados.

\section{MÉTODO}

\subsection{Participantes}

Un atleta de nivel nacional en carreras de montaña que participó voluntariamente en esta investigación (edad: 25,3 años; altura: $172 \mathrm{~cm}$; peso: $67 \mathrm{~kg}$; masa muscular: $59.1 \mathrm{~kg}$; masa grasa: $2.3 \mathrm{~kg}$; VO $\mathrm{V}_{\text {MAX: }} 70.2 \mathrm{ml} / \mathrm{kg} / \mathrm{min}$; FC REPOSO: 48 ppm; FC $\mathrm{FAX}_{\text {: }} 198$ $\mathrm{ppm}$ ). El atleta presentó una velocidad aeróbica máxima de $20.2 \mathrm{~km} / \mathrm{h}$ y una experiencia superior a tres años en la realización de esta modalidad deportiva. El 
atleta fue informado previamente de los detalles de la investigación y de sus posibles riesgos y beneficios, por lo que para ello se les suministró un consentimiento informado siguiendo el código de ética de la Asociación Médica Mundial (Declaración de Helsinki, 2013).

\subsection{Procedimiento}

La presente investigación se desarrolló durante la temporada 2016-2017, en una prueba de carácter federado dentro del calendario de pruebas oficiales de montaña organizada por la Federación de Montañismo de la Región de Murcia (FMRM). La carrera contaba con un desnivel positivo de 973 metros y con una distancia total del recorrido de $27.6 \mathrm{~km}$. El punto más alto de la prueba se encontraba a 1207 metros y el punto más bajo a 565 metros. En la figura 1 se representa el perfil completo de la prueba y la división de esta en distintos segmentos según la orografía para facilitar el análisis descriptivo del perfil, usando como criterio para la determinación de los distintos desniveles el aumento, descenso o mantenimiento del nivel de altura con respecto al mar. Los segmentos de la prueba son: segmento 1 (sin desnivel 1); segmento 2 (desnivel positivo 1); segmento 3 (sin desnivel 2); segmento 4 (desnivel positivo 2); segmento 5 (desnivel negativo 1); y segmento 6 (desnivel negativo 2 ).

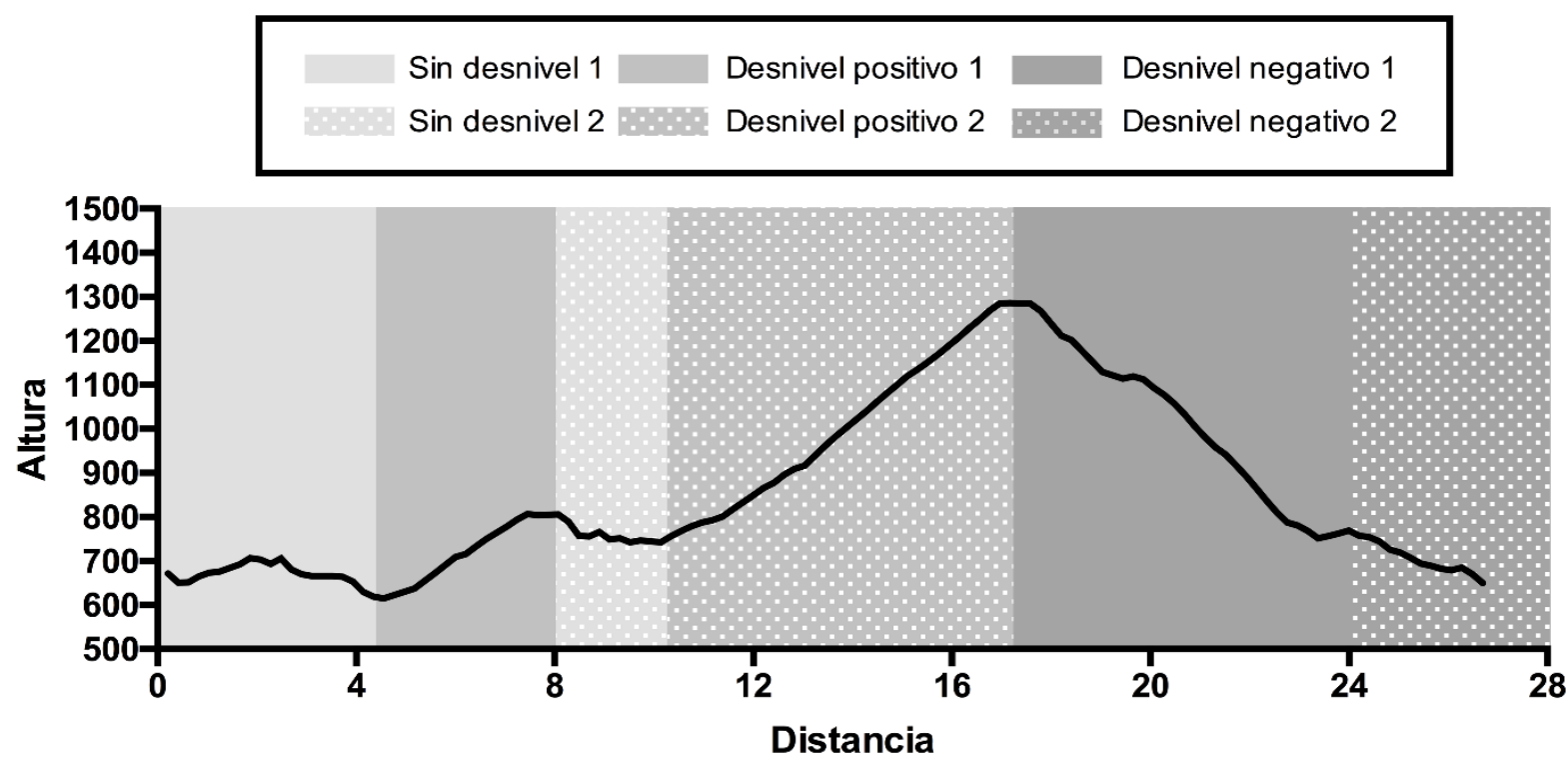

Figura 1. Perfil de la prueba y división en los 6 segmentos analizados en función de la pendiente. Eje $y$ (altura), eje $x$ (tiempo).

El protocolo de recogida de información se realizó durante tres sesiones en dos semanas. La primera sesión se realizó una evaluación de las características antropométricas del sujeto y la explicación acerca de los objetivos y la monitorización del atleta durante el estudio. La segunda sesión se realizó una familiarización con alta monitorización durante una prueba incremental máxima en tapiz rodante para evaluar el rendimiento del deportista. Finalmente, la segunda semana se realizó el registro de la prueba oficial de carrera de montaña. Para el 
registro de la segunda y tercera sesión, se citó al deportista 15 minutos previo al inicio de las pruebas para la colocación del material.

El dispositivo inercial (WIMU PRO ${ }^{T M}$, RealTrack Systems, Almería, España) se introdujo en un arnés que posteriormente se colocaba al atleta a la altura entre la segunda y cuarta vértebra torácica ajustado anatómicamente. Previamente el dispositivo fue autocalibrado a través de un proceso en la configuración interna del arranque. Para el autocalibrado se tuvo en cuenta tres aspectos: (i) dejar el dispositivo inmóvil durante $30 \mathrm{~s}$, (ii) situarlo en una superficie plana y (iii) $\sin$ dispositivos magnéticos alrededor (Bastida-Castillo, Gómez-Carmona, y PinoOrtega, 2016). Los datos fueron almacenados en la memoria interna del dispositivo, siendo posteriormente descargados y analizados mediante el software S PRO ${ }^{\mathrm{TM}}$ (RealTrack Systems, Almería, España).

\subsection{Variables}

En la presente investigación, a fin de que los resultados pudiesen compararse entre segmentos debido a la diferente distancia y tiempo empleado para completarse, las variables seleccionadas para el análisis de la carga física soportada fueron relativizadas al tiempo de ejecución, analizándose por minuto o calculando la media de exigencias de cada segmento.

\section{Carga externa}

- Player Load por minuto (PL/min): Es la suma vectorial de las aceleraciones del dispositivo en sus 3 ejes (vertical, anteroposterior y lateral). Esta variable se ha utilizado para evaluar la carga neuromuscular (Gómez-Carmona, BastidaCastillo, González-Custodio, Olcina, \& Pino-Ortega, 2019; Reche-Soto, CardonaNieto, Diaz-Suarez, Gómez-Carmona, \& Pino-Ortega, 2020). Se representa en unidades arbitrarias (a.u.) y se calcula a partir de la siguiente ecuación:

$$
\begin{gathered}
P L_{n}=\sqrt{\frac{\left(X_{n}-X_{n-1}\right)^{2}+\left(Y_{n}-Y_{n-1}\right)^{2}+\left(Z_{n}-Z_{n-1}\right)^{2}}{100}} \\
\text { PL acumulado }=\sum_{n=0}^{m} P L_{n} \times 0,01
\end{gathered}
$$

- Power metabolic por minuto (PM/min): Es el resultado de la multiplicación de la velocidad $(V$ por el coste energético de la actividad $(C E)$ derivado de la inclinación y la aceleración (Osgnach, Poser, Bernardini, Rinaldo, \& Di Prampero, 2010; Reche-Soto et al., 2019). Se representa en W/kg y es calculada a través de la siguiente fórmula:

$$
P M e t=C E \cdot V
$$


- Velocidad (V): Relación que se establece entre el espacio o la distancia (d) que recorre un objeto y el tiempo $(t)$ que invierte en ello. Se calcula a través de la siguiente fórmula:

$$
V=d \cdot t^{-1}
$$

- Entropía (ApEn): La entropía aproximada (ApEn) es una medida que depende de la probabilidad condicional de que dos secuencias que son similares para $m$ muestras permanezcan similares, dentro de una tolerancia $r$, en la próxima muestra $m+1$. Si una secuencia de datos contiene una gran cantidad de patrones repetitivos (predecible o más regular) tendrá una ApEn pequeña, mientras que una con pocos (menos predecible o más irregular) tendrá una ApEn mayor (Pincus, 2001). Para una serie temporal $\{x(\mathrm{n})\}$ de $\mathrm{N}$ muestras finitas, y definidos los parámetros $r$ y $m$, ApEn $(m, r, N)$ se puede calcular mediante la siguiente ecuación (aplicada sobre la señal del acelerómetro):

$$
\operatorname{ApEn}(m, r, N)=\operatorname{ApEn}(m, r)=\phi^{m}(r)-\phi^{m+1}(r)
$$

- Vertical stiffness o fuerzas de reacción vertical durante la carrera (KVERT): Es la relación existente entre la resultante de la fuerza máxima aplicada $\left(F_{\max }\right)$ y el desplazamiento vertical del centro de masas $\left(\Delta y_{c}{ }^{-1}\right)$ (Morin, Dalleau, Kyröläinen, Jeannin, y Belli, 2005) y se calcula a partir de la siguiente fórmula:

$$
k_{\text {vert }}=F_{\max } \cdot \Delta y_{c}{ }^{-1}
$$

\section{Carga interna}

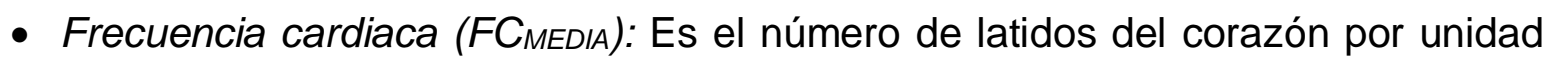
de tiempo. Se mide en pulsaciones por minuto y es una clara respuesta fisiológica al aumento de la actividad (Bouzas, Ottoline, y Delgado, 2010).

\subsection{Instrumentos}

\section{Características antropométricas}

La altura del sujeto fue medida con un tallímetro de pared (SECA, Hamburgo, Alemania). El peso corporal, masa muscular y masa grasa del atleta se obtuvo mediante una báscula de composición corporal compuesto por 8 electrodos de contacto modelo BC-601 (TANITA, Tokio, Japón).

\section{Carga externa}

La adquisición de los datos se llevó a cabo empleando un dispositivo inercial denominado WIMU PRO'M (RealTrack Systems, Almería, España). El dispositivo 
dispone diferentes sensores (4 acelerómetros, tres giroscopios, un magnetómetro, un chip GPS y otro UWB, entre otros). Para el registro de las variables velocidad y $\mathrm{PM} /$ min se utilizó el sensor de posicionamiento global por satélite (GPS) con una frecuencia de muestreo de $10 \mathrm{~Hz}$, siendo su fiabilidad y validez evaluada previamente (Bastida-Castillo, Gómez-Carmona, De la Cruz Sánchez, y PinoOrtega, 2018). Para el registro de las variables $\mathrm{PL} / \mathrm{min}$ y KVERT se utilizaron los 4 acelerómetros que componen el dispositivo, con un fondo de escala de $\pm 16 \mathrm{~g}, \pm 16 \mathrm{~g}$, $\pm 32 \mathrm{~g}$ y $\pm 400 \mathrm{~g}$, los cuales registraron a una frecuencia de muestreo de $100 \mathrm{~Hz}$, siendo evaluada su fiabilidad en una investigación previa (Gómez-Carmona, Bastida-Castillo, García-Rubio, Ibáñez, \& Pino-Ortega, 2019).

\section{Carga interna}

Se registró mediante el empleo de una banda GARMIN ${ }^{\top M}$ (Garmin Ltd., Olathe, Kansas, Estados Unidos) que se colocaba al sujeto justo por debajo de la apófisis xifoides, debajo de la línea intermamaria, la cual sincronizaba los datos al sistema WIMU PRO ${ }^{T M}$ a través de tecnología Ant+ con una frecuencia de muestreo de $4 \mathrm{~Hz}$, siendo los datos almacenados en el dispositivo. Este protocolo ha sido evaluado previamente (Molina-Carmona, Gomez-Carmona, Bastida-Castillo, y Pino-Ortega, 2018).

\section{Análisis estadístico}

Los datos de las variables analizadas se muestran como promedios y desviaciones estándar (promedio $\pm D E$ ) para describir todas las exigencias o requerimientos realizados durante la prueba. El análisis de la distribución de los datos se realizó mediante la prueba Shapiro-Wilk (Field, 2013), obteniendo una distribución normal. Se realizó una comparación ANOVA de un factor entre los segmentos de la prueba, utilizando el post-hoc de Bonferroni para la comparación por pares. Para calcular la magnitud de las diferencias entre segmentos se utilizó el estadístico $d$ de Cohen, siendo clasificado según Hopkins, Marshall, Batterham y Hanin (2009)como: efecto bajo $(0-0.2)$, efecto pequeño $(0.2-0.6)$, efecto moderado $(0.6-1.2)$, efecto grande $(1.2-2.0)$ y efecto muy grande $(>2.0)$. Los gráficos fueron realizados mediante el software Graphpad Prism (Graphpad Software Inc., La Jolla CA, EEUU). Las pruebas estadísticas fueron realizadas mediante el software SPSS 24.0 (SPSS Inc., Chicago IL, EEUU). La significación estadística se estableció con el valor de $\mathrm{p}<$ 0.05 .

\section{RESULTADOS}

En la tabla 1 se muestra el análisis descriptivo de las variables analizadas en esta investigación en los diferentes segmentos la prueba en relación con la orografía del terreno. Se encuentran los mayores valores en las variables $\mathrm{PL} / \mathrm{min}, \mathrm{PM} / \mathrm{min}$, velocidad y KVERT en los segmentos sin desnivel, mientras que los mayores valores en $\mathrm{FC}_{\mathrm{MEDIA}}$ se hallaron en los segmentos con desnivel positivo. 
Tabla 1. Media y desviación estándar de las variables analizadas en función del segmento de la prueba.

\begin{tabular}{|c|c|c|c|c|c|c|c|c|c|c|c|c|}
\hline \multirow[t]{2}{*}{ Segmento } & \multicolumn{2}{|c|}{$\begin{array}{l}\mathrm{PL} / \mathrm{min} \\
\text { (u.a.) }\end{array}$} & \multicolumn{2}{|c|}{$\begin{array}{c}\mathrm{PM} / \mathrm{min} \\
(\mathrm{W} / \mathrm{kg})\end{array}$} & \multicolumn{2}{|c|}{$\begin{array}{c}\mathrm{FC}_{\text {MEDIA }} \\
\text { (ppm) }\end{array}$} & \multicolumn{2}{|c|}{$\begin{array}{c}\text { Velocidad } \\
(\mathrm{km} / \mathrm{h})\end{array}$} & \multicolumn{2}{|c|}{$\begin{array}{l}\text { ApEn } \\
\text { (u.a.) }\end{array}$} & \multicolumn{2}{|c|}{$\mathrm{K}_{\mathrm{VERT}}$} \\
\hline & $M$ & $\mathrm{DE}$ & $M$ & $\mathrm{DE}$ & $M$ & $\mathrm{DE}$ & $M$ & $\mathrm{DE}$ & $M$ & $\mathrm{DE}$ & $M$ & $\mathrm{DE}$ \\
\hline SD 1 & 4.45 & 1.11 & 16.29 & 1.63 & 182.72 & 5.64 & 15.58 & 1.53 & 0.52 & 0.07 & 29.31 & 2.84 \\
\hline DP 1 & 3.37 & 0.76 & 13.35 & 1.36 & 191.11 & 1.31 & 12.85 & 1.31 & 0.49 & 0.05 & 26.01 & 1.54 \\
\hline SD 2 & 4.20 & 0.68 & 15.30 & 1.50 & 185.21 & 3.60 & 14.28 & 1.64 & 0.48 & 0.04 & 28.91 & 1.97 \\
\hline DP 2 & 2.78 & 0.60 & 10.34 & 2.18 & 189.33 & 1.70 & 9.82 & 1.99 & 0.46 & 0.05 & 25.25 & 1.49 \\
\hline DN 1 & 4.00 & 0.44 & 13.38 & 1.22 & 178.65 & 3.10 & 12.47 & 1.17 & 0.49 & 0.06 & 27.70 & 2.00 \\
\hline DN 2 & 3.78 & 0.64 & 14.06 & 1.69 & 186.52 & 2.83 & 13.47 & 1.72 & 0.51 & 0.04 & 27.31 & 1.01 \\
\hline Total & 3.76 & 0.71 & 13.79 & 1.60 & 185.59 & 3.03 & 13.08 & 1.56 & 0.49 & 0.05 & 27.41 & 1.81 \\
\hline
\end{tabular}

Nota. SD: Sin desnivel; DP: Desnivel positivo; DN: Desnivel negativo; PL/min: Player Load por minuto (unidad arbitraria); PM/min: Power Metabolic por minuto (watios/kilo); FC media (pulsaciones); ApEn: Entropía Aproximada (unidad arbitraria); Kvert: Índice de rigidez (unidad arbitraria); M: Media; DE: Desviación estándar.

Posteriormente, en la figura 2, se muestra la representación gráfica y el análisis comparativo de las variables analizadas en función del segmento al que pertenecen según el tipo de inclinación del terreno. En las variables $\mathrm{PL} / \mathrm{min}, \mathrm{PM} / \mathrm{min}$, Velocidad y Kvert se evidenciaron los mayores valores $(p<0.05)$ en los segmentos sin desnivel y los menores valores en los segmentos con desnivel positivo con un tamaño del efecto de moderado a muy grande (PL/min: $d=1.82-1.15 ; \mathrm{PM} / \mathrm{min}: d$ $=3.09$ - 1.36; Velocidad: $d=3.24-0.96$; KVERT: $d=1.79-1.64)$. En cambio, en la variable $\mathrm{FC}_{\text {MEDIA }}$ encontramos los mayores valores en los segmentos con desnivel positivo. Por último, en la variable ApEn se obtuvieron valores semejantes sin diferencias significativas $(p>0.24)$ en todos los segmentos analizados encontrando el valor más bajo en el segmento de desnivel positivo 2 .

En el análisis pormenorizado por segmentos en cuanto a las variables $\mathrm{PL} / \mathrm{min}$, KVERT y Velocidad se encontró mayores valores en los tramos sin desnivel $(p<0.05)$, posteriormente los tramos con desnivel negativo y finalmente los tramos con desnivel positivo que son los que reportan los menores valores en esta variable. Existen diferencias significativas en función del tipo de inclinación $(p<0.05)$, pero no entre los segmentos con el mismo tipo de desnivel $(p=0.23-0.68)$, excepto en la variable velocidad entre el segmento con desnivel positivo 1 y 2 con un tamaño del efecto grande $(p<0.05 ; d=1.79)$.

En la variable PM no se aprecian diferencias significativas entre los segmentos sin desnivel y los desniveles negativos. Únicamente el segmento con desnivel positivo 2 tuvo diferencias significativas con el resto de los segmentos $(p<0.01)$. Finalmente, en cuanto a la $\mathrm{FC}_{\text {MEDIA }}$ encontramos el menor valor en el segmento con desnivel negativo 1 (178.65 $\pm 3.10 \mathrm{ppm}$ ) y los mayores valores en los segmentos con desnivel positivo $(\mathrm{DP} 1=191.11 \pm 1.31 \mathrm{ppm}$; DP2 $=189.33 \pm 1.70 \mathrm{ppm})$, reportando diferencias con el resto de los segmentos $(p<0.05)$. No se encuentran diferencias entre los segmentos sin desnivel y el segmento con desnivel negativo 2 . 


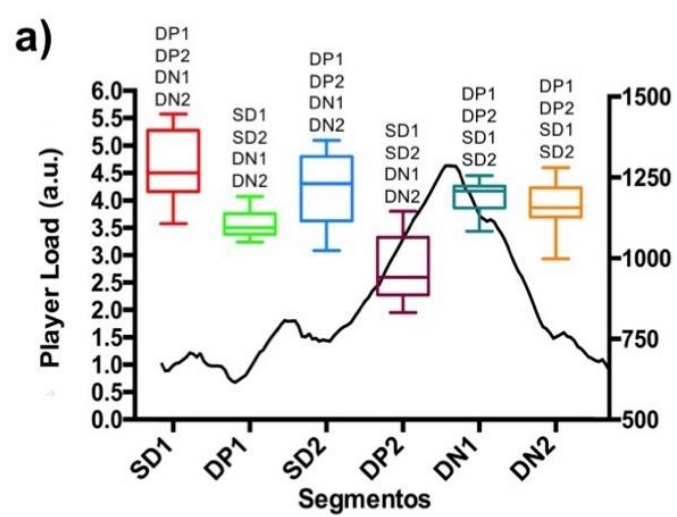

c)

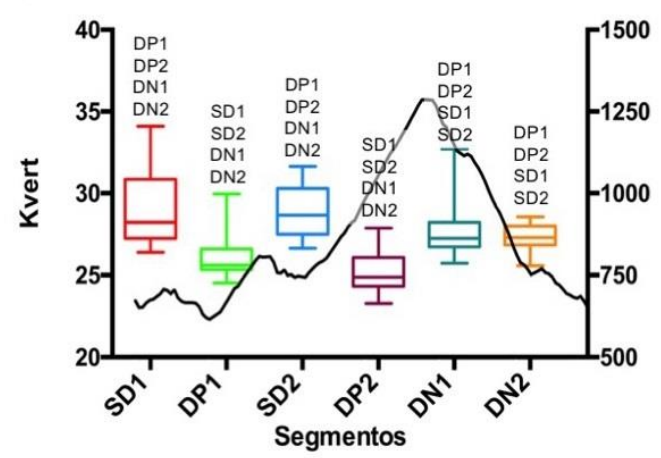

e)

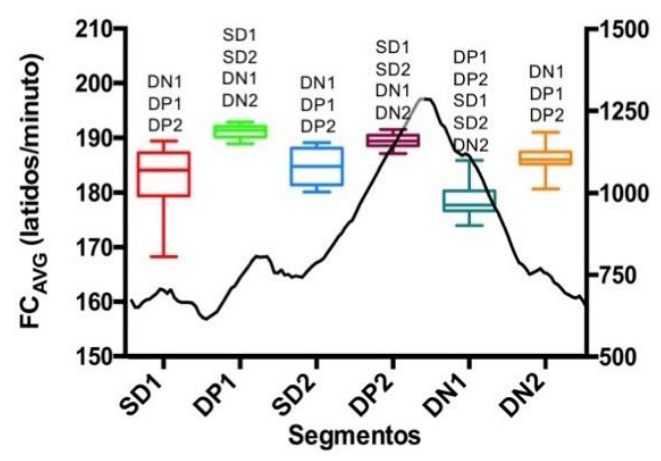

b)

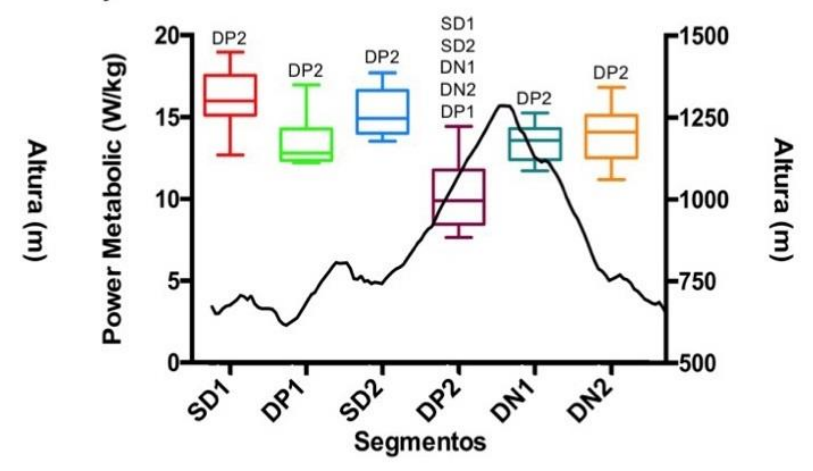

d)
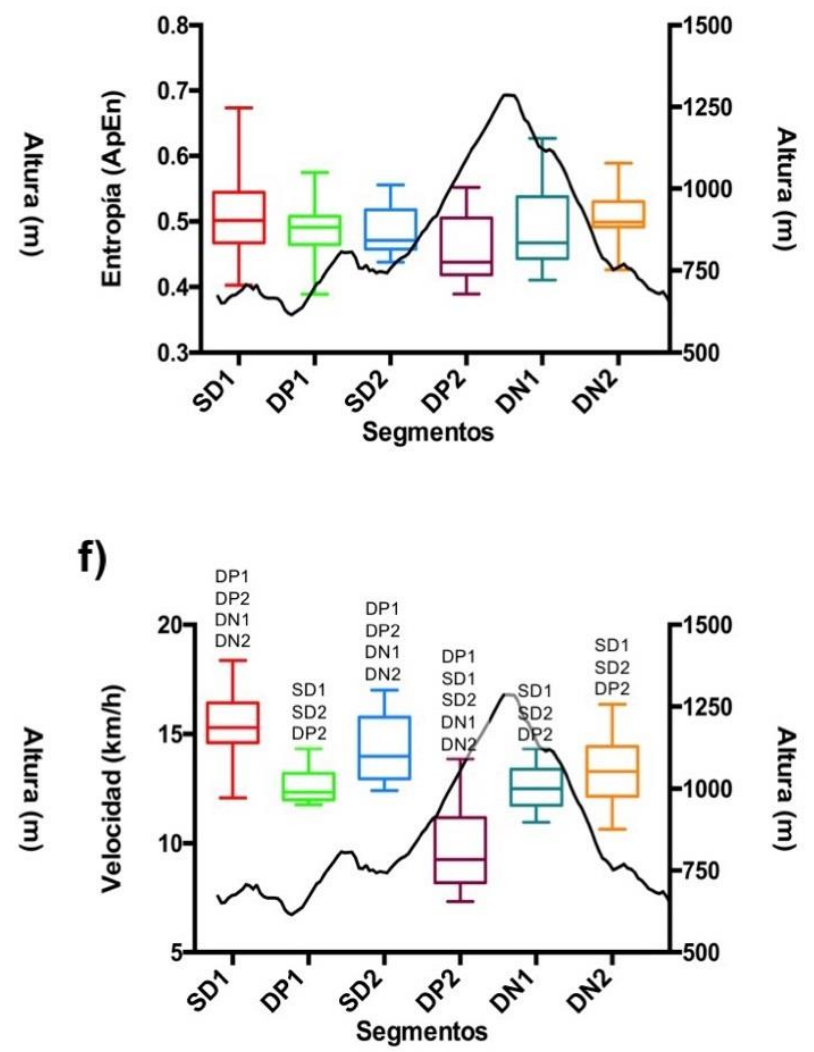

Figura 2. Gráfico de cajas y bigotes y diferencias significativas entre las variables en función de los segmentos diferenciados por la orografía del terreno: (a) Player Load (PL), (b) Power Metabolic (PM), (c) Stiffness vertical (KVERT), (d) Entropía aproximada (ApEn), (e) Frecuencia cardíaca $\left(F C_{M E D I A}\right)$ y (f) Velocidad.

SD1: Diferencias significativas respecto al segmento sin desnivel $1(p<0.05)$

SD2: Diferencias significativas respecto al segmento sin desnivel $2(p<0.05)$ DP1: Diferencias significativas respecto al segmento desnivel positivo $1(p<0.05)$ DP2: Diferencias significativas respecto al segmento desnivel positivo $2(p<0.05)$ DN1: Diferencias significativas respecto al segmento desnivel negativo $1(p<0.05)$ DN2: Diferencias significativas respecto al segmento desnivel negativo $2(p<0.05)$ 


\section{DISCUSIÓN}

Los objetivos de la presente investigación fueron describir la carga interna y externa durante una carrera de montaña y analizar la influencia del desnivel de la prueba en esta carga. A partir de los resultados obtenidos, se ha podido determinar que la carga a la que está sometida el sujeto varía dependiendo del tipo de desnivel que encuentre durante la carrera (Vernillo et al., 2017), al cual ha de adaptarse variando los factores mecánicos y fisiológicos.

En relación con la variable $\mathrm{PL} / \mathrm{min}$, se encuentran mayores valores en los segmentos con desnivel negativo y sin desnivel con relación a los tramos de desnivel positivo, siendo estas diferencias significativas $(p<0.05)$. Los estudios que analizan este parámetro están relacionados con los deportes colectivos donde las situaciones cambiantes dificultan la posibilidad de cuantificar la carga (Sparks, Coetzee, y Gabbett, 2017). De los resultados de estos se observa que el valor de $\mathrm{PL} / \mathrm{min}$ aumenta donde hay cambios significativos de velocidad, producto tanto de una aceleración como de una deceleración. Se ha visto también que las zonas donde las velocidades son más altas, el PL/min es mayor (Barrett et al., 2014; Buchheit, Gray, y Morin, 2015). Por lo tanto, un mayor PL/min en los segmentos con desnivel negativo está asociado a los cambios de velocidades significativos que se dan por las aceleraciones/deceleraciones (Sparks, Coetzee y Gabbett, 2017) y a mayores impactos producidos por los desniveles negativos (Vernillo et al., 2017). En cambio, los altos valores en los segmentos sin desnivel están asociados a un incremento de la intensidad de los desplazamientos registrada mediante la velocidad (Barrett, Midgley y Lovell, 2014; Nedergaard et al., 2017). Por tanto, esta variable podría ser un indicador adecuado para el análisis de las exigencias debido a su alta sensibilidad en función de la pendiente y por lo tanto su capacidad para identificar cambios en la mecánica de carrera provocada, por ejemplo por la velocidad (Barreira et al., 2017; Barrett et al., 2014; Nedergaard et al., 2017) y fatiga (Barrett et al., 2016; Cormack et al., 2013), entre otros.

En el caso del PM, los resultados muestran que los valores más altos se reportaron en las zonas de mayor velocidad que correspondían con las zonas sin desnivel (Osgnach et al., 2010). El consumo más bajo se encontró en los dos segmentos de desnivel positivo donde los valores de velocidad alcanzada fueron más bajos. Estos resultados se encuentran en contraste con la bibliografía, ya que la mayor parte de los estudios encontrados reconocen un mayor gasto energético en los tramos de subida (Vernillo et al., 2015; 2017), donde se requieren una mayor demanda de aplicación de fuerza (Gottschall y Kram, 2005). Se requiere de más información para ver su capacidad de detectar los cambios en el consumo energético debido al desnivel, ya que dicho cálculo está optimizado para estimación en deportes colectivos donde la superficie de trabajo es plana.

En cuanto a la $\mathrm{FC}_{\mathrm{MEDIA}}$, los resultados mostraron una gran variabilidad, dándose los datos más altos en las zonas de desnivel positivo $(\mathrm{DP} 1=191.1 \pm 1.31 \mathrm{ppm}$; DP2 = $189.33 \pm 1.70 \mathrm{ppm})$. Todas las diferencias fueron significativas $(p<0.05 ; d=0.52$ - 
2.05) exceptuando las que se desprendían de la comparación entre el tramo sin desnivel 2 y el tramo con desnivel negativo $2(p=0.37)$. Esto contrasta con lo recogido por Born, Stöggl, Swarén, y Björklund, (2017) donde no encontraron diferencias en la $\mathrm{FC}_{\text {MEDIA }}$ en función del tipo de orografía, si encontrando estas en la saturación de oxígeno muscular $\left(\mathrm{SmO}_{2}\right)$. Por otro lado, los resultados de la FC se encuentran en consonancia con Chatterjee et al. (2015) donde se muestra que los cambios de pendiente afectaron directamente al $\mathrm{VO}_{2}$ y al gasto cardiaco siendo más altos en las zonas con un desnivel positivo. Por lo cual, la FC es un buen indicador de la dificultad física durante la prueba, detectando con precisión la dificultad de los segmentos con inclinación positiva, pero no diferenciando entre los segmentos sin inclinación y con inclinación negativa.

Las velocidades alcanzadas durante la carrera también variaron dependiendo del tipo de orografía del terreno, siendo mayores en los tramos donde no había desnivel. Las diferencias de velocidad entre los tramos sin desnivel y los de subida se deben, como se ha visto en distintos estudios (Born et al., 2017; Chatterjee et al., 2015) a una mayor demanda energética y un mayor consumo de oxígeno para mantener la intensidad. Por otro lado, la reducción de la velocidad en las zonas de desnivel negativo respecto a los segmentos sin desnivel se ha visto relacionada con el nivel técnico del corredor y la inclinación de la pendiente (Kay, 2014). De los resultados obtenidos para la ApEn, esta variable analiza la complejidad de la señal del dato bruto del acelerómetro, encontrado variaciones dependiendo del tipo de segmento, aunque no siendo estas significativas.

Finalmente, en la variable stiffness muscular (KVERT) se encuentra que en los segmentos de desnivel positivo los valores fueron más bajos que en el resto de segmentos, siendo todas las diferencias significativas. Esto concuerda con el trabajo de Giovanelli et al. (2016), donde se demostró que la fatiga en subida disminuye la capacidad para aplicar fuerza máxima, lo que afecta de forma directa al KVERT. Además, también se encontraron diferencias significativas entre los segmentos sin desnivel con los segmentos con desnivel negativo los cuales pudieron verse afectados por la fatiga acumulada durante el resto de la prueba ya que el estudio de Vernillo et al. (2015) demostró que el coste energético post carrera aumentaba en la bajada caracterizado por un menor tiempo de vuelo y un mayor número de pasos que demuestran una menor capacidad de aplicación de fuerza.

\section{CONCLUSIONES}

1. La carga externa e interna difiere significativamente durante la carrera de montaña entre los diferentes segmentos que compone la prueba en función del desnivel.

2. La pendiente positiva provoca una mayor carga interna registrada mediante FC MEDIA, mientras que los segmentos sin desnivel provoca una mayor carga externa registrada mediante $\mathrm{PL} / \mathrm{min}, \mathrm{PM} / \mathrm{min}$ y KVERT. La mayor velocidad se encontró en los tramos sin desnivel. 
3. La variable velocidad de desplazamiento y las variables de carga externa registradas (PM/min, PL/min y $\left.\mathrm{K}_{\mathrm{VERT}}\right)$ mostraron una dinámica similar en los segmentos analizados.

4. Las variables $\mathrm{PL} / \mathrm{min}$ y $\mathrm{K}_{\mathrm{VERT}}$ se encontraron como las variables útiles para el análisis de las exigencias de las carreras de montaña debido a su alta sensibilidad para discriminar los diferentes tipos de pendiente (positiva, negativa y sin pendiente).

\section{APLICACIONES PRÁCTICAS Y PROPUESTAS DE INVESTIGACIÓN}

La utilización de los sistemas inerciales, así como las variables medidas representativas de los cambios de orografía del terreno, pueden dar una visión más acertada de las exigencias en las carreras de montaña. Y así, a partir de esta información, diseñar entrenamientos específicos en base a la carga real en competición para evitar lesiones y sobre-entrenamiento.

La falta de estudios en torno a esta materia y la dificultad de la cuantificación del entrenamiento de esta modalidad por la variabilidad del terreno y las dispares demandas mecánicas y fisiológicas que se dan, hace necesaria una mayor investigación sobre la temática del estudio. Futuras investigaciones deberían seguir esta línea de análisis modificando la secuencia de desniveles, así como el grado de pendiente de estos para tratar de determinar cómo afectan en el rendimiento. Además, parece necesario el análisis con un mayor número de participantes y de competiciones para comparar la carga del corredor tanto intra-sujeto en diferentes competiciones como inter-sujetos dentro de una misma competición.

Es necesario ampliar la evidencia científica de la influencia de la fatiga, deshidratación, temperatura ambiental, e impacto neuromuscular, entre otras condiciones presentes en este tipo de pruebas de fondo y ultra fondo en la variabilidad de la carga externa e interna.

\section{REFERENCIA BIBLIOGRÁFICA}

Banister, E. W. (1991). Modeling elite athletic performance. En H.J. Green, J.D. McDougar y H. Wenger (Eds.) Physiological Testing of Elite Athletes (pp. 403424). Champaign, Illinois: Human Kinetics.

Barreira, P., Robinson, M. A., Drust, B., Nedergaard, N., Raja Azidin, R. M. F., y Vanrenterghem, J. (2017). Mechanical Player Load using trunk-mounted accelerometry in football: Is it a reliable, task- and player-specific observation? Journal of Sports Sciences, 35(17), 1674-1681. https://doi.org/10.1080/02640414.2016.1229015

Barrett, S., Midgley, A., y Lovell, R. (2014). PlayerLoad ${ }^{\mathrm{TM}}$ : Reliability, Convergent Validity, and Influence of Unit Position during Treadmill Running. International 
Journal of Sports Physiology and Performance, 9(6), 945-952. https://doi.org/10.1123/ijspp.2013-0418

Barrett, S., Midgley, A. W., Towlson, C., Garrett, A., Portas, M., y Lovell, R. (2016). Within-Match PlayerLoad ${ }^{\mathrm{TM}}$ Patterns during a Simulated Soccer Match: Potential Implications for Unit Positioning and Fatigue Management. International Journal of Sports Physiology and Performance, 11(1), 135-140. https://doi.org/10.1123/ijspp.2014-0582

Bastida-Castillo, A., Gómez-Carmona, C. D., De la Cruz Sánchez, E., y Pino-Ortega, J. (2018). Accuracy, intra- and inter-unit reliability, and comparison between GPS and UWB-based position-tracking systems used for time-motion analyses in soccer. European Journal of Sport Science, 18(4), 450-457. https://doi.org/10.1080/17461391.2018.1427796

Bastida-Castillo, A., Gómez-Carmona, C. D., y Pino-Ortega, J. (2016). Efectos del Tipo de Recuperación Sobre la Oxigenación Muscular Durante el Ejercicio de Sentadilla. Kronos, 15(2), 1-13.

Born, D.-P., Stöggl, T., Swarén, M., y Björklund, G. (2017). Near-Infrared Spectroscopy: More Accurate Than Heart Rate for Monitoring Intensity in Running in Hilly Terrain. International Journal of Sports Physiology and Performance, 12(4), 440-447. https://doi.org/10.1123/ijspp.2016-0101

Borresen, J., y Lambert, M. I. (2009). The quantification of training load, the training response and the effect on performance. Sports Medicine, 39(9), 779-795. https://doi.org/10.2165/11317780-000000000-00000

Bouzas, J. C., Ottoline, N. M., y Delgado, M. (2010). Aplicaciones de la frecuencia cardiaca máxima en la evaluación y prescripción de ejercicio. Apunts. Medicina le l'Esport, 45(168), 251-258. https://doi.org/10.1016/j.apunts.2010.04.003

Boyd, L. J., Ball, K., y Aughey, R. J. (2013). Quantifying external load in Australian football matches and training using accelerometers. Int $J$ Sports Physiol Perform, 8(1), 44-51. https://doi.org/10.1123/ijspp.8.1.44

Buceta, J. M., López de la Llave, A., Pérez-Llantada, M.C., Vallejo, M., y del Pino, M.D. (2007). Intervención psicológica con corredores de maratón: Características y valoración del programa aplicado en el maratón de Madrid. Revista de Psicología del Deporte, 11(1), 83-109.

Buchheit, M., Gray, A., y Morin, J.B. (2015). Assessing stride variables and vertical stiffness with GPS-embedded accelerometers: Preliminary insights for the monitoring of neuromuscular fatigue on the field. Journal of sports science \& medicine, 14(4), 698-701.

Chatterjee, T., Paul, S., Pramanik, A., Chowdhury, B., Pal, M. S., y Majumdar, D. (2015). Cardio-Respiratory and Metabolic Changes during Continuous UphillDownhill Load Carriage Task. Ergonomics for Rural Development, 3-10. Recuperado de: http://inet.vidyasagar.ac.in:8080/jspui/handle/123456789/193

Consejo Superior de Deportes. (2016). Encuesta sobre los hábitos deportivos en España (2015). Madrid: Consejo Superior de Deportes y Centro de Investigaciones Sociológicas.

Cormack, S. J., Mooney, M. G., Morgan, W., y McGuigan, M. R. (2013). Influence of 
neuromuscular fatigue on accelerometer load in elite Australian football players. International journal of sports physiology and performance, 8(4), 373-378. https://doi.org/10.1123/ijspp.8.4.373

Eich, T. S., y Metcalfe, J. (2009). Effects of the stress of marathon running on implicit and explicit memory. Psychonomic Bulletin \& Review, 16(3), 475-479. https://doi.org/10.3758/PBR.16.3.475

Field, A. (2013). Discovering Statistics Using IBM SPSS Statistics (4. ${ }^{a}$ ed.). Londres: SAGE.

Gabbett, T. (2013). Quantifying the Physical Demands of Collision Sports: Does Microsensor Technology Measure What It Claims to Measure? Journal of Strength and Conditioning Research, 27(8), 2319-2322. https://doi.org/10.1519/JSC.0b013e318277fd21

Giovanelli, N., Taboga, P., Rejc, E., Simunic, B., Antonutto, G., y Lazzer, S. (2016). Effects of an Uphill Marathon on Running Mechanics and Lower-Limb Muscle Fatigue. International Journal of Sports Physiology and Performance, 11(4), 522-529. https://doi.org/10.1123/ijspp.2014-0602

Gómez-Carmona, C. D., Bastida-Castillo, A., García-Rubio, J., Ibáñez, S. J., y PinoOrtega, J. (2019). Static and dynamic reliability of WIMU PROTM accelerometers according to anatomical placement. Proceedings of the Institution of Mechanical Engineers, Part P: Journal of Sports Engineering and Technology, 233(2), 238-248. https://doi.org/10.1177/1754337118816922

Gómez-Carmona, C. D., Bastida-Castillo, A., González-Custodio, A., Olcina, G., y Pino-Ortega, J. (2019). Using an inertial device (WIMU PRO ${ }^{\mathrm{TM}} \square$ ) to quantify neuromuscular load in running: Reliability, convergent validity and the influence of type of surface and device location. The Journal of Strength and Conditioning Research, 34(2), 365-373. https://doi.org/10.1519/JSC.0000000000003106

Gottschall, J. S., y Kram, R. (2005). Ground reaction forces during downhill and uphill running. Journal of Biomechanics, 38(3), 445-452. https://doi.org/10.1016/j.jbiomech.2004.04.023

Gutiérrez-Vargas, R., Martín-Rodríguez, S., Sánchez-Ureña, B., RodríguezMontero, A., Salas-Cabrera, J., Gutiérrez-Vargas, J. C., ... Rojas-Valverde, D. (2020). Biochemical and Muscle Mechanical Postmarathon Changes in Hot and Humid Conditions: Journal of Strength and Conditioning Research, 34(3), 847-856. https://doi.org/10.1519/JSC.0000000000002746

Gutiérrez-Vargas, R., Ugalde-Ramírez, J. A., Rojas-Valverde, D., Salas-Cabrera, J., Rodríguez-Montero, A., \& Gutiérrez-Vargas, J. C. (2017). La termografía infrarroja como herramienta efectiva para detectar áreas músculares dañadas después de correr una maratón. Revista de la Facultad de Medicina, 65(4), 601-607. https://doi.org/10.15446/revfacmed.v65n4.60638

Halson, S. L. (2014). Monitoring Training Load to Understand Fatigue in Athletes. Sports Medicine, 44(S2), 139-147. https://doi.org/10.1007/s40279-014-0253z

Hopkins, W. G., Marshall, S. W., Batterham, A. M., y Hanin, J. (2009). Progressive Statistics for Studies in Sports Medicine and Exercise Science: Medicine \& Science in Sports \& Exercise, 41(1), 3-13. 
https://doi.org/10.1249/MSS.0b013e31818cb278

Hughson, R. L., Weisiger, K. H., y Swanson, G. D. (1987). Blood lactate concentration increases as a continuous function in progressive exercise. Journal of Applied Physiology, 62(5), 1975-1981. https://doi.org/10.1152/jappl.1987.62.5.1975

Jobson, S. A., Passfield, L., Atkinson, G., Barton, G., y Scarf, P. (2009). The analysis and utilization of cycling training data. Sports medicine, 39(10), 833-844. https://doi.org/10.2165/11317840-000000000-00000

Kerhervé, H. A., Millet, G. Y., y Solomon, C. (2015). The Dynamics of Speed Selection and Psycho-Physiological Load during a Mountain Ultramarathon. PLOS ONE, 10(12), e0145482. https://doi.org/10.1371/journal.pone.0145482

Kyröläinen, H., Pullinen, T., Candau, R., Avela, J., Huttunen, P., y Komi, P. V. (2000). Effects of marathon running on running economy and kinematics. European Journal of Applied Physiology, 82(4), 297-304. https://doi.org/10.1007/s004210000219

Martin, V., Kerherve, H., Messonnier, L. A., Banfi, J. C., Geyssant, A., Bonnefoy, R., ... Millet, G. Y. (2010). Central and peripheral contributions to neuromuscular fatigue induced by a 24-h treadmill run. Journal of Applied Physiology, 108(5), 1224-1233. https://doi.org/10.1152/japplphysiol.01202.2009

Millet, G. (2011). Can Neuromuscular Fatigue Explain Running Strategies and Performance in Ultra-Marathons?: The Flush Model. Sports Medicine, 41(6), 489-506. https://doi.org/10.2165/11588760-000000000-00000

Millet, G., Martin, V., Lattier, G., y Ballay, Y. (2003). Mechanisms contributing to knee extensor strength loss after prolonged running exercise. Journal of Applied Physiology, 94(1), 193-198. https://doi.org/10.1152/japplphysiol.00600.2002

Millet, G. Y., Banfi, J. C., Kerherve, H., Morin, J. B., Vincent, L., Estrade, C., ... Feasson, L. (2011). Physiological and biological factors associated with a 24 $\mathrm{h}$ treadmill ultra-marathon performance: Factors associated with ultramarathon performance. Scandinavian Journal of Medicine \& Science in Sports, 21(1), 54-61. https://doi.org/10.1111/j.1600-0838.2009.01001.x

Millet, Guillaume, Tomazin, K., Verges, S., Vincent, C., Bonnefoy, R., Boisson, R.C., ... Martin, V. (2011). Neuromuscular Consequences of an Extreme Mountain Ultra-Marathon. PLoS ONE, 6(2), e17059. https://doi.org/10.1371/journal.pone.0017059

Millet, Guillaume Y., Morin, J.-B., Degache, F., Edouard, P., Feasson, L., Verney, J., y Oullion, R. (2009). Running from Paris to Beijing: Biomechanical and physiological consequences. European Journal of Applied Physiology, 107(6), 731-738. https://doi.org/10.1007/s00421-009-1194-3

Molina-Carmona, I., Gomez-Carmona, C. D., Bastida Castillo, A., y Pino-Ortega, J. (2018). Validez del dispositivo inercial WIMU $\mathrm{PRO}^{\mathrm{TM}} \square$ para el registro de la frecuencia cardíaca en un test de campo. Sport-TK: Revista Euroamericana de Ciencias del Deporte, 7, 81-86. https://doi.org/10.6018/321921

Morin, J. B., Tomazin, K., Edouard, P., y Millet, G. Y. (2011). Changes in running mechanics and spring-mass behavior induced by a mountain ultra-marathon race. Journal of Biomechanics, 44(6), 1104-1107. https://doi.org/10.1016/j.jbiomech.2011.01.028 
Morin, J. B., Dalleau, G., Kyröläinen, H., Jeannin, T., y Belli, A. (2005). A Simple Method for Measuring Stiffness during Running. Journal of Applied Biomechanics, 21(2), 167-180. https://doi.org/10.1123/jab.21.2.167

Morin, J. B., Samozino, P., y Millet, G. Y. (2011). Changes in Running Kinematics, Kinetics, and Spring-Mass Behavior over a 24-h Run: Medicine \& Science in Sports \& Exercise, 829-836. https://doi.org/10.1249/MSS.0b013e3181fec518

Nedergaard, N. J., Robinson, M. A., Eusterwiemann, E., Drust, B., Lisboa, P. J., y Vanrenterghem, J. (2017). The Relationship Between Whole-Body External Loading and Body-Worn Accelerometry During Team-Sport Movements. International Journal of Sports Physiology and Performance, 12(1), 18-26. https://doi.org/10.1123/ijspp.2015-0712

Nicol, C., Komi, P. V., y Marconnet, P. (2007). Effects of marathon fatigue on running kinematics and economy. Scandinavian Journal of Medicine \& Science in Sports, 1(4), 195-204. https://doi.org/10.1111/j.1600-0838.1991.tb00296.x

Osgnach, C., Poser, S., Bernardini, R., Rinaldo, R., y Di Prampero, P. E. (2010). Energy Cost and Metabolic Power in Elite Soccer: A New Match Analysis Approach. Medicine \& Science in Sports \& Exercise, 42(1), 170-178. https://doi.org/10.1249/MSS.0b013e3181ae5cfd

Pichot, V., Roche, F., Gaspoz, J.-M., Enjolras, F., Antoniadis, A., Minini, P., ... Barthelemy, J. C. (2000). Relation between heart rate variability and training load in middle-distance runners. Medicine and Science in Sports and Exercise, 32(10), 1729-1736. https://doi.org/10.1097/00005768-20001000000011

Pincus, S. M. (2001). Assessing serial irregularity and its implications for health. Annals of the New York Academy of Sciences, 954(1), 245-267. https://doi.org/10.1111/j.1749-6632.2001.tb02755.x

Reche-Soto, P., Cardona-Nieto, D., Diaz-Suarez, A., Bastida-Castillo, A., GomezCarmona, C., Garcia-Rubio, J., y Pino-Ortega, J. (2019). Player Load and Metabolic Power Dynamics as Load Quantifiers in Soccer. Journal of Human Kinetics, 69(1), 259-269. http://www.doi.org/10.2478/hukin-2018-0072

Reche-Soto, P., Cardona-Nieto, D., Diaz-Suarez, A., Gomez-Carmona, C., y PinoOrtega, J. (2020). AcelT y PlayerLoad ${ }^{T M} \square$ : Dos variables para la cuantificación de la carga neuromuscular. Revista Internacional de Medicina y Ciencias de la Actividad Física y del Deporte, 20(77), 167-183. https://doi.org/10.15366/rimcafd2020.77.011

Rojas-Valverde, D. (2019). Brief historical review of distance and ultradistance runnig in Costa Rica and the world. Viref:revista de Educación Física, 8(1), 1 19.

Ruiz-Juan, F., y Zarauz, A. (2014). Análisis de la motivación en corredores de maratón españoles. Revista Latinoamericana de Psicologia, 46(1), 1-11. https://doi.org/10.1016/S0120-0534(14)70001-9

Sparks, M., Coetzee, B., y Gabbett, T. J. (2017). Internal and External Match Loads of University-Level Soccer Players: A Comparison Between Methods. Journal of Strength and Conditioning Research, 31(4), 1072-1077. https://doi.org/10.1519/JSC.0000000000001560 
Taylor, K., Chapman, D., Cronin, J., Newton, M. J., y Gill, N. (2012). Fatigue monitoring in high performance sport: A survey of current trends. Journal of Australian Strength \& Conditioning, 20(1), 12-23.

Twist, C., y Highton, J. (2013). Monitoring fatigue and recovery in rugby league players. International Journal of sports physiology and performance, 8(5), 467-474. https://doi.org/10.1123/ijspp.8.5.467

Urdampilleta, A., López-Grueso, R., Martínez-Sanz, J. M., y Mielgo-Ayuso, J. (2014). Parámetros bioquímicos básicos, hematológicos y hormonales para el control de la salud y el estado nutricional en los deportistas. Revista Española de Nutrición Humana y Dietética, 18(3), 155-171. https://doi.org/10.14306/renhyd.18.3.24

Uth, N., Sorensen, H., Overgaard, K., y Pedersen, P. K. (2004). Estimation of VO2max from the ratio between HRmax and HRrest? The Heart Rate Ratio Method. European Journal of Applied Physiology, 91(1), 111-115. https://doi.org/10.1007/s00421-003-0988-y

Vernillo, G., Giandolini, M., Edwards, W. B., Morin, J.-B., Samozino, P., Horvais, N., y Millet, G. Y. (2017). Biomechanics and Physiology of Uphill and Downhill Running. Sports Medicine, 47(4), 615-629. https://doi.org/10.1007/s40279016-0605-y

Vernillo, G., Savoldelli, A., Zignoli, A., Skafidas, S., Fornasiero, A., La Torre, A., ... Schena, F. (2015). Energy cost and kinematics of level, uphill and downhill running: Fatigue-induced changes after a mountain ultramarathon. Journal of Sports Sciences, 33(19), 1998-2005. https://doi.org/10.1080/02640414.2015.1022870

Número de citas totales / Total references: 53 (100\%) Número de citas propias de la revista / Journal's own references: $1(1,88 \%)$

Rev.int.med.cienc.act.fís.deporte - vol. 20 - número 80 - ISSN: 1577-0354 